\title{
Haploidentical Related Peripheral Blood Stem Cell Transplantation as a Salvage Transplantation in Patients with Graft Failure
}

\author{
Shigeo Fuji \\ Key words: graft failure, haploidentical, hematopoietic stem cell transplantation
}

(Intern Med 54: 2709-2710, 2015)

(DOI: 10.2169/internalmedicine.54.5323)

Primary graft failure (GF) is a potentially lethal complication following an allogeneic hematopoietic cell transplantation (HCT). The risk of GF is not insignificant, with a rate as high as $10-20 \%$ after cord blood transplantation (CBT). Without autologous recovery, patients with GF need an urgent salvage HCT, as prolonged neutropenia is associated with a significant risk of severe infectious complications. Tachibana and colleagues reported a successful case of salvage HCT after GF (1). While modern transplant physicians are more experienced in such urgent HCT than previous doctors, the rescue of a patient with GF is still a difficult proposition. Although the best conditioning regimens or graft-versus-host disease (GVHD) prophylaxis have not yet been determined, there are several important considerations that can improve the outcome of salvage HCT for GF.

First, the choice of the stem cell source for salvage HCT is important. Patients who undergo CBT usually do not have a human leukocyte antigen (HLA)-matched related donor. Thus, CBT or a haploidentical related donor can be an alternative stem cell source as they are readily available. We have previously reported on a nationwide survey of the clinical outcome of 220 patients who underwent salvage HCT for GF after CBT (2). In that report, the most commonly used stem cell source was cord blood (180 patients). However, when using peripheral blood stem cells (PBSC) from a haploidentical donor, the engraftment was significantly faster and the engraftment rate was significantly higher, which led to a significantly better outcome in patients using a haploidentical HCT compared to those using a CBT. Thus, in general, PBSC transplantation from a haploidentical donor is considered the first choice in patients with primary GF. In stable patients, who might wait for the engraftment for at least two weeks, CBT might be also an option.
Second, the timing of the diagnosis of GF is important. Usually, GF is diagnosed when the neutrophil level does not recover, even at day 28 after allogeneic HCT. However, we do not have to wait until day 28 to diagnose GF. Although there is no clear evidence, if there is no sign of neutrophil recovery in the peripheral blood even in three weeks after CBT, the physicians have to think about the possibility of GF. The level of neutrophil recovery should be checked using a smear of peripheral blood. In such a case, chimerism analysis should be performed using peripheral blood or a bone marrow aspirate. If there is no detectable donor chimerism using these samples, the physician should start to prepare for salvage HCT. The earlier preparations begin, the earlier salvage HCT can be performed, which would be expected to result in a better outcome.

Third, it is also important to check HLA typing of relatives, including parents, children and even cousins, when available. We readily expect parents and children to be potential haploidentical donors, but the possibility of finding a haploidentical cousin is also high.

Fourth, the choice of the conditioning regimen can be an important factor. Recently, a short-term conditioning regimen, a so-called "one-day regimen," has been widely used $(3,4)$. Such a short conditioning regimen focusing on the immunosuppressive effects of using fludarabine plus cyclophosphamide with or without total body irradiation, is highly effective in achieving engraftment and is tolerable even in patients with GF.

If salvage HCT is conducted before a patient develops severe complications such as infection, we can still expect that a significant proportion of patients with GF can be rescued by salvage HCT. When patients with a high risk of GF receive HCT, the physicians have to determine in advance, how they rescue the patient in case of GF. 
The author states that he has no Conflict of Interest (COI).

\section{References}

1. Tachibana T, Yamamoto E, Kawasaki R, et al. Successful engraftment and durable graft-versus-host disease control with haploidentical peripheral blood and a short-term conditioning regimen for primary graft failure. Intern Med 54: 2749-2752, 2015.

2. Fuji S, Nakamura F, Hatanaka K, et al. Peripheral blood as a preferable source of stem cells for salvage transplantation in patients with graft failure after cord blood transplantation: a retrospective analysis of the registry data of the Japanese Society for Hematopoietic Cell Transplantation. Biol Blood Marrow Transplant 18: 1407-1414, 2012.

3. Sumi M, Shimizu I, Sato K, et al. Graft failure in cord blood transplantation successfully treated with short-term reducedintensity conditioning regimen and second allogeneic transplantation. Int J Hematol 92: 744-750, 2010.

4. Shimizu I, Kobayashi H, Nasu K, et al. Successful engraftment of cord blood following a one-day reduced-intensity conditioning regimen in two patients suffering primary graft failure and sepsis. Bone Marrow Transplant 44: 617-618, 2009.

(C) 2015 The Japanese Society of Internal Medicine http://www.naika.or.jp/imonline/index.html 